Titre : Les origines de l'hésitation face à la vaccination

Auteur: André Picard

Publication : La santé publique à une ère marquée par le doute - Origines religieuses et culturelles de l'hésitation des Canadiens face à la vaccination

Directeurs : Paul Bramadat, Maryse Guay, Julie A. Bettinger et Réal Roy

Pages : $307-313$

ISBN : 978-2-7622-0359-2

URI : http://hdl.handle.net/11143/16032

DOI : https://doi.org/10.17118/11143/16032

Éditeur : Les Éditions de l'Université de Sherbrooke (ÉDUS) 


\title{
Les origines de l'hésitation face à la vaccination
}

\author{
ANDRÉ PICARD
}

Si seulement tous les parents avaient accès aux plus récentes études sur la vaccination... si seulement ils connaissaient mieux les efforts séculaires des autorités de santé publique pour contrer les maladies contagieuses fatales... si seulement les médias veillaient à informer le public au sujet des bienfaits réels et des risques de la vaccination, au lieu d'y semer la confusion...

On ne compte plus les «si seulement... » dans ce domaine. La recherche médicale nous a donné le pouvoir de prévenir beaucoup de maladies contagieuses de l'enfance, sinon la plupart, mais les virus et les bactéries peut-être fatales continuent de se propager et de causer des torts aux enfants à un rythme alarmant, parce que quelques parents hésitent à adopter des méthodes de prévention efficaces et abordables telle la vaccination.

Une cuiller à café comble de connaissances scientifiques, un peu de connaissances historiques et une généreuse pincée de plaidoyers dans les médias : serait-ce là la formule magique pour mettre un terme à ces hésitations?

Mais non, ce serait trop simple.

Cet ouvrage nous a permis d'éclaircir au moins un fait : la réticence et la vaccination sont nées au même moment. Autrefois, on refusait l'inoculation contre la variole; aujourd'hui, le vaccin contre le VPH subit les contrecoups - et la controverse se poursuivra malgré la validité des résultats de la recherche, malgré les efforts d'éducation de la population et malgré la dénonciation, dans les médias, des allégations trompeuses qui vont à l'encontre des messages de santé publique.

Une des erreurs les plus communes, en fait, consiste à faire de la réticence à la vaccination l'œuvre d'un seul «mouvement anti-vaccination » unifié et bien organisé. En réalité, bien que les différentes formes de la réticence se ressemblent quelque peu, les raisons pour lesquelles les parents sont réticents ou s'opposent à l'immunisation de leurs enfants sont nombreuses et de nature tant sociale que politique, culturelle ou religieuse. 
Cet ouvrage met en lumière la variété des craintes - réelles, imaginaires, religieuses et culturelles - et, par voie de conséquence, l'ampleur du défi à relever. Les partisans de la vaccination ne devraient peut-être pas s'attendre à ce que la science supplante la foi de fidèles ardents : les autorités de santé publique ne gagneront probablement jamais la confiance des personnes à qui les institutions publiques inspirent une méfiance profonde et l'intérêt de tous ne sera sans doute jamais la priorité de ceux pour qui la liberté individuelle a la préséance.

Devraient-ils alors abandonner?

Bien sûr que non.

Les autorités de santé publique et les praticiens de la médecine doivent s'entêter à promouvoir la réduction des méfaits causés par la maladie. II n'y a pas de solution unique et simple aux craintes que soulève la vaccination. II faut plutôt établir un ensemble de réponses qui sont adaptées aux préoccupations d'auditoires variés, tout en reconnaissant qu'il est impossible de les apaiser complètement. Les groupes favorables à la vaccination - dont la motivation peut varier, comme celle des opposants - ne doivent ménager aucun effort pour réduire au minimum les torts causés aux individus et à la population en général; leur tâche consiste également à trouver des solutions aux nouveaux problèmes, comme la dissémination facile d'une information erronée par l'intermédiaire des médias sociaux.

Il faut également que les partisans de la vaccination veillent à considérer le problème en perspective. À peine 2 p. cent des parents refusent toute vaccination et approximativement 85 à 90 p. cent des enfants entrant à la maternelle sont au moins partiellement vaccinés.

Ce sont les gestes ou l'inaction de 10 à 15 p. cent de la population qui nuisent à l'immunité collective et permettent la réapparition de la rougeole, des oreillons et de la coqueluche, maladies de l'enfance pourtant en grande partie éliminées dans les pays occidentaux.

Le comportement du groupe des hésitants (qui, répétons-le, n'est pas un mouvement cohérent et organisé) est principalement motivé par l'angoisse. Les parents qui sont réticents à la vaccination de leurs enfants souhaitent réellement prendre la meilleure décision pour eux, mais ils en sont venus, pour différentes raisons, à croire que la vaccination comporte plus de risques que la maladie elle-même. On voit une grande confusion parmi leurs préoccupations : les vaccins ne pourraient-ils pas contenir des "poisons » comme le mercure, le système immunitaire de l'enfant pourrait-il être incapable d'accepter autant de vaccins (environ deux douzaines d'injections jusqu'à l'âge de deux ans) et qui voudrait infliger une telle souffrance à un enfant (en fait, rien de plus qu'une injection) pour le protéger contre un ennemi invisible?

Bon nombre de parents hésitants croient sincèrement que la vaccination n'est pas nécessaire parce qu'ils n'ont jamais, personnellement, vu ces maladies évitables par la vaccination autrefois si courantes. N'oubliez pas qu'il n'a fallu qu'une génération pour que la rougeole passe de 350000 cas par année à, jusqu'à récemment, quelques rares cas importés de l'étranger. D'une certaine façon, on pourrait donc dire que les formidables succès de la vaccination comptent parmi les principaux obstacles à l'acceptation de son maintien.

Nombreux sont aussi, parmi ces parents, ceux qui ont l'impression - sincère également qu'il se trouve d'autres moyens efficaces de remplacer les vaccins conventionnels injectés dans le bras. Les médecines que l'on qualifie d'alternatives et complémentaires (phytothérapie, homéopathie, chiropratique, naturopathie, acupuncture, etc.) sont plus populaires que 
jamais. Ce mouvement est en partie attribuable à la « chimiophobie » (une peur excessive ou déraisonnable des produits chimiques) et au concept erroné en vertu duquel tout ce qui est « naturel » (y compris les bactéries et les virus) ne peut faire de tort, contrairement à tout ce qui est fabriqué (comme les vaccins).

Toutes ces attitudes sont encore le résultat de la méfiance, notamment contre les grands fabricants de médicaments et les gouvernements autoritaires. Nous savons, hors de tout doute, que le secteur pharmaceutique a fait des erreurs et commis des excès, mais cela ne devrait pas nous amener à douter des bienfaits de tous les produits qu'il fabrique. Comme Bettinger et MacDonald l'expliquent au chapitre 8, nul ne peut nier l'efficacité et la rentabilité des vaccins pédiatriques.

Il faut aussi reconnaître que bon nombre de produits " alternatifs » sont extrêmement rentables pour leur fabricant, sans être assujettis à la même réglementation. Si de nombreux produits de cette catégorie, vendus pour remplacer les vaccins (comme les nosodes, qui sont les « vaccins » homéopathiques), sont devenus la cible des organismes de réglementation, ce n'est pas qu'il s'agit d'une conspiration de large portée, mais plutôt parce que leurs fabricants font à leur sujet des allégations démesurées qui ne sont guère appuyées par des données probantes.

On ne peut pas ignorer, également, que les médias ont joué un rôle considérable dans l'amplification de ces craintes et croyances; au mieux, on pourra les accuser d'avoir semé la confusion et l'incompréhension. L'opposition à la vaccination est une opinion minoritaire, mais nombreux sont ceux que cette forme de scepticisme attire à cause de ce qu'ils lisent dans les journaux, sur Internet et dans les médias sociaux ou entendent à la télévision.

Prenez le cas d'Andrew Wakefield, de triste mémoire, dont il a été question dans plusieurs chapitres du présent ouvrage et qui a publié dans The Lancet, en 1998, un article où il alléguait qu'il existait un lien entre le vaccin RRO (rougeole, rubéole, oreillons) et l'autisme, un trouble du développement. À ce moment, le nombre d'enfants vaccinés augmentait, tout comme l'incidence de l'autisme : on pouvait faire une association, mais non créer un lien causal. Cet article (dont on a démontré qu'il était fondé sur des données erronées et qui a depuis lors été retiré du périodique) déclencha une controverse (ou peut-être, plus précisément, une «frénésie médiatique ») qui dura pendant près de quinze ans et fit des dommages incalculables.

En termes scientifiques, le débat fut très court: la théorie selon laquelle les vaccins causaient l'autisme n'a jamais été crédible et encore moins biologiquement plausible. Les efforts que Wakefield a faits dans son propre intérêt (car il avait conçu un vaccin contre la rougeole qui devait remplacer le vaccin RRO et le rendre riche) ont servi de réponse à la question qui hantait les parents (« Pourquoi y a-t-il de plus en plus de cas d'autisme? »), en proposant une explication - la vaccination - que nombre d'entre eux ont trouvé providentielle.

Les craintes que suscitait la vaccination sont nées bien avant la « recherche » de Wakefield, mais elles ont contribué à soulever la «tempête parfaite » qui a servi de prétexte facile à des millions de personnes depuis lors devenues des adversaires de la vaccination. Dans les faits, toute l'histoire des vaccins comme causes de l'autisme a mené bien des gens à réfléchir de nouveau à la notion d'objectivité, notamment en ce qui concerne les rapports en matière de santé. Curtis Brainard, en 2013, après une analyse approfondie de la couverture médiatique de l'affaire Wakefield, écrivait ce qui suit dans le Columbia Journalism Review: «Dans de tels cas, 
il peut être irresponsable d'assurer une couverture «équilibrée », puisqu'on laisse supposer une controverse, alors qu'il n'y en a pas. »

Un équilibre trompeur peut faire de grands torts, notamment dans le signalement des cas problèmes en santé. Les journalistes ont pour tâche de distinguer les données probantes de celles qui n'ont aucun sens; il ne suffit pas de rester au-dessus de la mêlée en discourant sur l'équilibre, surtout quand la santé des enfants est en jeu. En toute justice, nous reconnaissons que de nombreux journalistes ont joué un rôle de leader et ont puissamment défendu la vaccination (et ont parfois été de meilleurs porte-parole que les scientifiques), dont Seth Mnookin (auteur de The Panic Virus [2011]) et Ben Goldacre (auteur de la populaire chronique Bad Science, dans The Guardian), mais ces gens ne peuvent à eux seuls contrer le flux constant d'anecdotes alarmistes et de pseudo-reportages qui dominent souvent les débats au sujet de la vaccination. Les médias doivent se responsabiliser et veiller à ne rapporter que la vérité, surtout dans les situations cruciales où le public se tourne vers eux pour se guider.

Il faut reconnaître également que l'affaire Wakefield s'est produite en même temps que des changements profonds transformaient le milieu médiatique. Le développement des réseaux sociaux fait en sorte qu'il est plus facile que jamais de propager des messages trompeurs vers un auditoire élargi; il est aussi plus simple de confirmer des partis pris erronés et d'influencer grandement les comportements liés à la santé, de manière consciente ou inconsciente. La démocratisation de l'information a généralement eu un effet positif, mais elle a rendu beaucoup plus difficile la tâche de promouvoir les initiatives de santé publique - et la vaccination en particulier. Maintenant qu'Internet existe, il est possible de trouver des « preuves » confirmant et consolidant une conviction ou un préjugé et d'ainsi supplanter ce que dit la science.

Mais alors, quel est le meilleur moyen de s'assurer que le plus grand nombre possible d'enfants bénéficient de la vaccination et que la propagation des maladies de l'enfance est réduite au minimum, sinon éliminée?

La censure n'est pas la bonne réponse. Il est impossible de fermer tous les sites Web qui vantent l'opposition à la vaccination et de faire de même pour tous les comptes de médias sociaux où un père ou une mère exprime des doutes quant aux bienfaits ou à l'innocuité des vaccins.

Les scientifiques doivent au contraire s'appuyer sur la science. Il faut s'efforcer de rendre les vaccins plus sûrs et plus efficaces (on notera que de considérables améliorations ont été apportées au fil du temps) et l'administration des vaccins doit être rendue plus facile en éliminant la douleur (et les injections).

On doit aussi préparer des messages de riposte et tenter sans cesse d'imbriquer des données factuelles dans un débat souvent trop émotif, même si l'auditoire les reçoit sans grand enthousiasme. Pour chaque histoire d'enfant qui aura subi un tort à cause d'un vaccin, il faudra rappeler les dommages que les maladies contagieuses infligent à un nombre incalculable d'autres enfants. Ce sera une bataille de tous les instants, car on peut difficilement capter les sentiments du public en lui racontant l'histoire d'un enfant qui n'est jamais malade ou qui, ayant été vacciné, n'est pas mort. C'est malheureusement la tâche des autorités de santé publique. 
Si irritants que soient les hésitants pour les professionnels de la santé, l'approche autoritaire n'est pas la meilleure. Le but visé est de persuader, et non de condamner. Les poursuites judiciaires doivent être le dernier recours contre les parents - très peu nombreux, je le répète - qui sont réticents ou s'opposent tout simplement à la vaccination. II faut respecter les convictions religieuses, sans toutefois oublier qu'il existe d'autres droits. Refuser la vaccination ne doit pas se faire facilement. À tout le moins, il faut offrir aux parents hésitants des conseils rappelant l'importance de l'immunisation pour leurs propres enfants et pour la population en général. Les convictions culturelles qui sont anormales et déraisonnables (voire suprarationnelles, comme le dit Bramadat ailleurs dans cet ouvrage) ne doivent pas permettre que certains enfants soient exemptés des immunisations préalables à l'entrée à l'école et il ne faut pas les imposer aux professionnels de la santé exerçant à l'hôpital ou dans d'autres milieux de soins.

Sur le plan personnel, les pédiatres et les médecins de famille devraient personnellement entamer un dialogue avec les parents réticents, plutôt que de les abandonner à leur sort. À l'échelle de la société, les autorités de santé publique devraient être prêtes à engager le combat directement avec les opposants à la vaccination. Depuis trop longtemps, elles se sont gardées d'agir, prétextant qu'en se lançant dans le débat, elles donneraient une certaine crédibilité à un groupe marginal, mais bruyant. L'inaction n'a pas fonctionné, malheureusement; elle a simplement fait naître une culture d'incertitude qui a grandement facilité la vie des personnes qui reportent ou refusent la vaccination.

En matière scientifique, il n'y a plus de doute : la vaccination fonctionne. Elle possède beaucoup d'avantages, contre peu de risques. Ce qui reste à choisir - et ce ne sera pas une tâche facile -, c'est le meilleur moyen de motiver la vaste majorité de la population pour qu'elle adhère à ce message, ce qui revient souvent à faire mieux connaître des concepts scientifiques complexes comme ceux des bienfaits et des risques et à entamer des dialogues au sujet des origines religieuses et culturelles de la réticence à la vaccination comme nous l'avons fait dans le présent ouvrage.

Une communication de qualité - c'est-à-dire élaborer un message et le communiquer de manière claire et uniforme - est l'arme la plus puissante de l'arsenal de santé publique.

La réticence à la vaccination ne se décline pas en noir et blanc. Cet ouvrage nous aidera à en comprendre la complexité et à saisir la diversité des convictions et craintes, ainsi que leur évolution au fil du temps, le cas échéant. C'est aussi un rappel du fait que la meilleure manière de ne pas répéter les erreurs du passé consiste à bien le connaître. N'est-il pas bizarre de revoir les arguments du docteur Dagenais, qui s'opposait à la vaccination contre la variole en 1875, dans les allégations du docteur Mercola qui demandait la cessation de la vaccination contre la rougeole en 2014 ? Plus ça change ...

Le plus puissant message que transmet cet ouvrage se lit entre les lignes : les défenseurs de la vaccination ont toujours la même bataille à livrer parce qu'ils ont à de nombreuses reprises négligé de saisir des occasions incomparables - notamment à chaque éclosion importante d'une maladie contagieuse - de promouvoir plus vigoureusement la vaccination et, plus largement, la santé des enfants. Ils ne peuvent pas se permettre d'oublier la puissance de la vaccination, ni se résigner au fait qu'il y aura toujours des réticents. Ce serait vraiment déplorable si, dans quelques années, ils jetaient un regard sur le passé en se disant : " Si seulement nous avions fait plus... » 


\section{BIBLIOGRAPHIE}

Brainard, Curtis. 2013. "Sticking with the Truth: How 'Balanced' Coverage Helped Sustain the Bogus Claim that Childhood Vaccines Can Cause Autism." Columbia Journalism Review (May/June). http://www. cjr.org/feature/sticking_with_the_truth.php.

Mnookin, S. 2011. The Panic Virus: A True Story of Medicine, Science, and Fear. New York: Simon and Schuster. 